\title{
Mechanics of materially uniform thin films
}

Steigmann, David, dsteigmann@berkeley.edu, University of California

\begin{abstract}
A rigorous pure-bending model for thin films, accurate to leading order in film thickness, is established on the basis of the three-dimensional theory of materially uniform elastic bodies. An extension of the model, accounting for both stretching and bending and incorporating a generalization of Koiter's theory, is proposed. One conclusion is that the classical Kirchhoff-Love hypothesis, i.e., the assumption that material lines normal to the film remain so in the course of deformation, does not obtain. The model is applicable to materials that have undergone prior plastic deformation, diffusion, or growth.
\end{abstract}

